Slave boson large degeneracy expansion for the lattice Anderson model (invited)

A. J. Millis, M. Lavagna, and P. A. Lee

Citation: Journal of Applied Physics 61, 3904 (1987); doi: 10.1063/1.338578

View online: https://doi.org/10.1063/1.338578

View Table of Contents: http://aip.scitation.org/toc/jap/61/8

Published by the American Institute of Physics

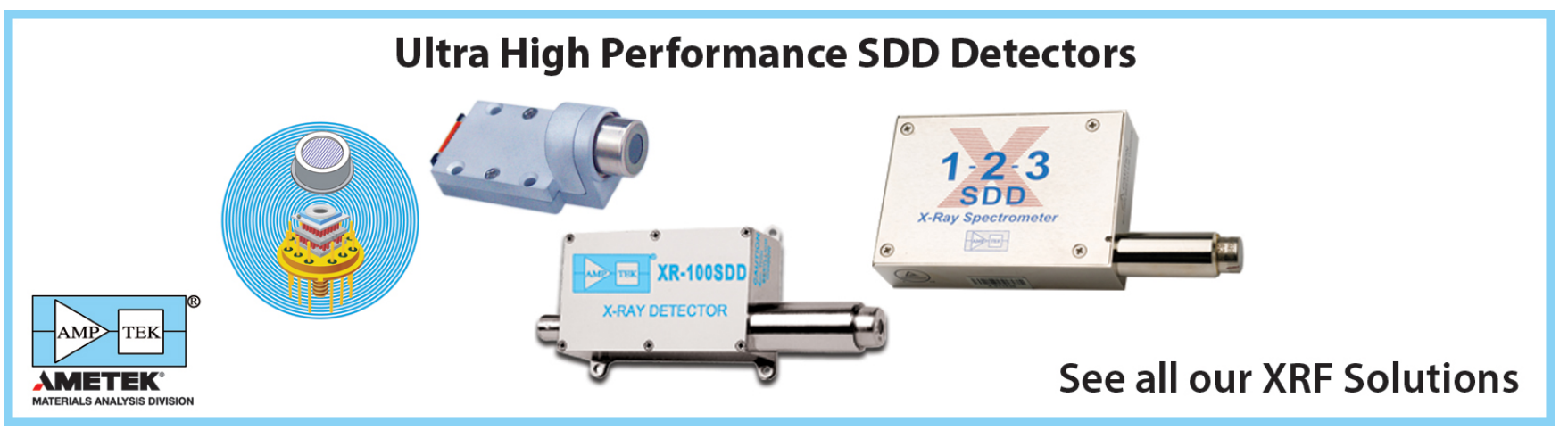




\title{
Slave boson large degeneracy expansion for the lattice Anderson model (invited)
}

\author{
A. J. Millis, ${ }^{\text {a) }}$ M. Lavagna, ${ }^{\text {b) }}$ and P. A. Lee \\ Department of Physics, Massachusetis Institute of Technology, Cambridge, Massachusetts 02139
}

\begin{abstract}
The slave boson formalism will be reviewed, a comparison of Kondo spin compensation in the impurity and in the lattice will be given, results for the temperature- and frequency-dependent conductivity will be presented, the density-density correlation function will be discussed, and a calculation of the slave-boson mediated $d$-wave superconducting instability will be outlined. Throughout, we emphasize the differences between the lattice and one impurity Anderson modeis and the strengths and weaknesses of the slave boson method.
\end{abstract}

In this paper we discuss some aspects of the slave boson large degeneracy $(N)$ approach to the low-temperature properties of the $U=\infty$ lattice Anderson model, in the Kondo limit, in which it is believed to describe the currently interesting heavy fermion materials. For a review of other approaches to the Anderson model and for a justification of the applicability of this model to the heavy fermion materials, see Ref. 1. The slave boson method was originally applied to the single impurity Anderson model. ${ }^{2,3}$ In this paper we shall discuss its application to the latice problem. We first review the formalism and the central approximations of the method, comparing the lattice and impurity problems. Then we give a few applications of the method: to the question of the Kondo spin compensation in the lattice, to the frequency- and temperature-dependent conductivity $\sigma(\omega, T)$, to the density luctuation collective mode, and to the superconducting instability. Throughout we discuss the relation of our results to those of the single impurity problem and we outline the implications for understanding the ground state of the heavy fermion materials. The conclusion is an attempt to assess the utility of the slave boson method for the heavy fermion problem. This paper summarizes work done by the authors over the past year. Details and further applications are presented elsewhere., The slave boson technique has been applied to the lattice problem by other authors. ${ }^{6-8}$. Some of this work, especially the elegant Fermiliquid approach of Auerbach and Levin, ${ }^{7}$ will be referred to below, but a detailed review will not be presented.

The $U=\infty$ Anderson model describes a structureless band of conduction electrons (operator $c_{k \sigma}$, energy $\epsilon_{k}$ ) hybridizing with localized $f$ electrons (operator $f_{i m}$, energy $E_{0}$ ) subject to the constraint that the number of felectrons on site $i, n_{f} \leqslant 1$. The hybridization takes place via a term in the Hamiltonian which may be schematically written $\left(V e^{i k R_{i}} c_{k \sigma}^{+} f_{i m}+\right.$ H.c.). $V$ is the hybridization matrix element, usually assumed to be structureless. Because the mod-

\footnotetext{
"Current address: A.T.\&T. Bell Laboratories, 600 Mountain Ave, Murray Hill, NJ 07974.

b) Current address: Laboratoire Lowis Neel, C. N. R. S., 166x 38042 Grenoble Cedex, France.
}

el includes $c-f$ hybridization, it does not conserve $n_{f}^{i}$ : the inequality constraint is thus difficult to implement. One way to proceed is to use the slave boson method ${ }^{2,3,9}$ in which one introduces a new boson field $b_{i}^{+}$, which creates the state in which the $f$ orbitals on site $i$ are unoccupied, and rewrites the hybridization as $\left(V e^{i k R_{i}} c_{k: f}^{+} f_{i m} b_{i}^{+}+\right.$H.c.), and the constraint as

$$
Q_{i}=\sum_{m} f_{i m}^{+} f_{i m}+b_{i}+b_{i}=1
$$

As long as one stays within the mainfold of states which are eigenstates of $Q_{i}$ with eigenvalue 1 , the Hamiltonian has the same matrix eiements as the original Anderson model, ${ }^{2}$ but it may be attacked by conventional field theoretical techniques, including a $1 / N$ expansion.

To understand the $1 / N$ expansion, consider first the one-impurity problem, in which the sum on lattice sites $i$ in Eq. ( 1 ) is restricted to a single site, conventionally taken to be the origin. One then writes the $f$ states in a basis of total angular momentum $J$. Usually only one value of $J$, say $J_{f}$, is relevant, and $m$ indexes the $z$ components of $J$. One expands the conduction electrons in the same way and finds that all $c$ electrons with $J=J_{f}$ decoupie, so that one is left with a problem in which $N=2 J_{f}+1$ degenerate bands of $c$ electrons hybridize with $N$ degenerate $f$ levels ${ }^{10} ;$ a $1 / N$ expansion results, yielding curves which quantitatively agree with experimental data on dilute alloys of Ce in a La host. ${ }^{11}$ This expansion is not available in the lattice problem because $J$ is not conserved as $c$ electrons propagate from site to site. However, in the slave boson work so far this complication has been ignored, and it has been assumed that both $c$ and $f$ electrons can be characterized by an $N$-fold degenerate spin quantum number $m$, with $-J_{f} \leqslant m \leqslant J_{f}$ and $N=2 J_{f}+1 . m$ is taken to be conserved both in $c \cdot f$ hybridization and in propagation through the lattice. Also, for computational convenience, the $c$-electrons are taken to have a free-electron dispersion relation and a spherical Fermi surface. Umilapp processes are neglected. The consequences of these drastic approximations are not known; it is hoped that the resulting model yields results which are qualitatively similar to the physically realistic case. It is unclear if the simplified model can be quantitatively compared with experiment; qualitative agreement is in many cases good. If the assumptions are 
granted, though, the model possesses a $1 / N$ expansion similar to that found in the single impurity problem.

To perform the $1 / N$ expansion one writes down the Hamiltonian described above, introduces a Lagrange multiplier field $\lambda$ to enforce the constraint (1), and then decomposes $b$ and $\lambda$ into mean-field and fluctuating parts. The decomposition may be formulated in either the radial or the Cartesian gauge. In the radial gauge one writes $b_{i}=r_{i} e^{i \theta_{i}}$, defines a new $f$ operator, $f^{\prime}$, via $f_{i}=f_{i}^{i} e^{i \theta_{i}}$, and rewrites the model in terms of $r, \theta$, and $f^{\prime}$. Use of the radial garge necessitates a Lagrangian functional integral formulation; we will write the model in the Cartesian version here for simplicity. The radial gauge formulation is, however, more convenient for many computations because infrared divergences occur in the Cartesian gauge which must be carefnlly cancelled from physical quantities. ${ }^{412}$ One obtains the same results in either formulation. In the Cartesian gauge one writes $b=\sqrt{N}(a+A)$ where $a$ is the mean field and $A$ the fluctuating part of $b$. After making the decomposition one finds that the Hamiltonian $H$ may be written $H=B_{0}+H_{I}+H_{A}$, where

$$
\begin{aligned}
H_{0}= & \sum_{k m} \epsilon_{k} c_{k m}^{+} c_{k m}+\epsilon_{f} f_{k m}^{+} f_{k m}+V a\left(c_{k m}^{+} f_{m}+H . c_{.}\right) \\
& +N \sum_{k}\left(\epsilon_{f}-E_{0}\right) A_{k}^{+} A_{k s} \\
H_{I}= & V \sum_{k q m}\left(c_{k m}^{+} f_{k+q m} A_{q}^{+}+H . c .\right) \\
& +\sum_{k q} \lambda_{q}\left(N A_{k+q}^{+} A_{k}+\sum_{m} f_{k+q m}^{+} f_{k m}\right) \\
H_{A}= & N\left(\epsilon_{f}-E_{0}\right) a\left(A_{k=0}^{+}+A_{k=0}\right)+N\left(a^{2}-q_{0}\right) \lambda .
\end{aligned}
$$

$E_{0}$ is the original $f$-level energy. We are incerested in the Kondo limit in which $\eta=-E_{0} / \rho_{0} V^{2} \geqslant 1 . \rho_{0}=d k / d \epsilon_{k}$ is the $c$-electron density of states. $E_{f}$ is the mean field and $\lambda$ the fluctuating part of the original Lagrange multiplier field.

The mean-field parameters are fixed as follows: the "anomalous" terms in $H_{A}$ lead to "tadpole" graphs, in which a boson line terminates at a point, requiring that all tadpoles vanish fixes $a$ and $\epsilon_{f}{ }^{4}$ To leading order in $N$ one finds ( $W$ is cefined in Fig. 1):

$$
\begin{aligned}
& \epsilon_{f}=W e^{\varepsilon_{0} / \rho_{0} V^{2}}, \\
& a^{2}=q_{0} \epsilon_{f} / \rho_{0} V^{2} .
\end{aligned}
$$

$\epsilon_{f}$ satisfies the same equation as (and is to be identified

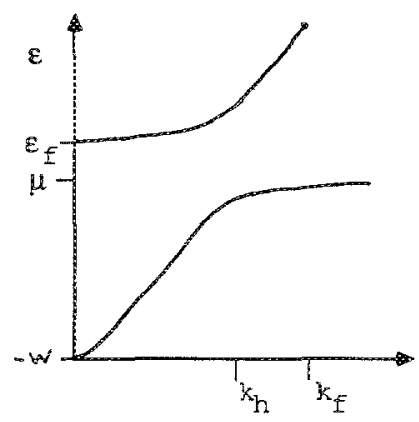

FIG. 1. Sketch of energy $\epsilon$ vs wavenumber $k$ disperson telation for the quasiparticle bands derived from $H_{0}$. A less than half-filled band is assumed. The magnitude of the smallest direct gap, which occurs at $k=k_{h}$, is $2 \% a$. with) the one-impurity Kondo temperature $T_{K} \cdot \epsilon_{f}$ is the smallest energy scale in the problem. It is also the effective Fermi temperature of the heavy fermions.

As can be seen from $H_{0}$, Eq. (2a), the parameter $a$ renormalizes the hybridization $V$. By Eq. (3b), in the Kondo limit $a \ll 1$.

We comment on the parameter $q_{0}$ which appears in Eqs. (2c) and (3b). According to our conventions the constraint (1) may be written $n_{f}^{i}=N\left(q_{0}-a^{2}\right)$. Our model is only equivalent to the original Anderson model if $Q_{0}=1 / N$, but to obtain a sensible $1 / N$ expansion we must regard $q_{0}$ as a parameter indeperdent of the explicit $N$ in Eqs. (2), and only set $q_{0}=1 / N$ at the end of any calculation. Otherwise, as Eq. (3b) shows, $a$ would be of order $1 / N$ and to leading order in $1 / N$ the hybridization would vanish, and the formalism would not describe the coherence effects found in a Kondo lattice. This problem does not occur in the impurity case, when one may set $q_{0}=1 / N$ at the outset. ${ }^{3}$ For our procedure to be sensible, physical quantities must be analytic functions of $q_{0}$; while this seems a priori implausible, considering the singular effects of setting $a=0$ in Eq. (2a), the various calculations we have performed yield expressions for physical quantities which have a smooth limit as $q_{0} \rightarrow 0$, indicating that the procedure is sensible. In particular, for thermodynamic quantities we find ${ }^{4}$ (in agreement with previous work ${ }^{6}$ ) that the leading order results are those of a scaled-up one-impurity model in which the density of impurities is set equal to the density of $f$ sites in the lattice. Other authors advocate treating $q_{0}$ as an independent parameter ${ }^{7,12}$; the relation of the resulting "generalized Anderson model" to the physical Anderson model is unclear.

$H_{0}$, Eq. (2a), describes a mean-nield theory of noninteracting electrons moving in a band structure determined by the new $f$-level energy $\varepsilon_{f}$ and the renormalized hybridization $V a$. This band structure is sketched in Fig. 1. The Fermi momentum $k_{F}$ is fixed by the total number of electrons, as usual. We have assumed that the Fermi level is in the lower band, equivalent results are obtained if it is in the upper band. Note that the band is very fiat at $k=k_{F}$ : the lowenergy excitations are heavy fermions of mass $m^{*}=(\mathrm{VQ})$ $\left.\varepsilon_{f}\right)^{2} m$, where $m$ is the $c$-electron band mass. The electronic excitations of this band structure are to be interpreted as quasiparticles; the Fermi energy for these quasiparticles is $\epsilon_{f}{ }^{4}$ It is sometimes asserted that the mean-field theory is exact as $N \rightarrow \infty$; this staternent must be interpreted with caution. It is true that to leading order in $1 / N, \gamma=\left(m^{*} / m\right) \gamma_{0}$ and $\chi=\left(m^{*} / m\right) \chi_{0}$, where $\gamma_{0}$ and $\chi_{0}$ are the specific-heat coefficient and susceptibility that the $c$ electrons alone would have. ${ }^{4,6,7}$ However, for density fluctuations, phenomena involving energies comparable to or greater than $\epsilon_{f}$, transport quantities and the $f$ spectral weight, even the leading order results are not those of noninteracting electrons moving in the band structure shown in Fig. $1{ }^{4}$

Corrections to the mean field theory may be thought of as coming from the electron-boson interactions described by $H_{I}$. It turns out that $1 / N$ is the coupling constant for these interactions; thus, the $1 / N$ expansion is essentially a perturbation theory in the electron-boson interaction. Within this expansion, one can compute essentially anything desired. 
We now discuss some resutits of such calculations.

We first consider Kondo spin compensation in the impurity and in the lattice. In the context of the one-impurity Anderson model it has been argued ${ }^{13}$ that in the $T=0$ ground state there exists a spin-polarization cloud which surrounds the impurity, is made of $c$ electrons with energies within $T_{K}$ of the Fermi surface, has size $\xi_{0}=v_{F} / T_{K}$, and is locked together with the impurity spin to form a singlet. It is clear that this picture cannot be extended to a lattice: there are not enough $c$ electrons within $T_{K}$ of the Fermi surface to compensate every $f$ spin. We have computed (to leading order in $1 / N) \chi_{c f}(r)=\left\langle M_{f}^{z}(0, t) M_{c}^{z}(p, t)\right\rangle$, the correlation function between the $z$ component of an $f$ spin at the origin and the conduction electron spin at point $r$, for both the oneimpurity and lattice Anderson models. In the one-impurity case $\chi_{c f}$ has precisely the features described above: the dominant contribution comes from $c$ electrons with energies within $T_{K}$ of the Fermi surface, the range is $\xi_{0}$ (for $r>\xi_{0}, \chi_{c f}$ falls of as $r^{-4}$, and the integral over $r$ of $\chi$ shows it contains one $c$ electron. In the lattice case the relevant energy scale is $V G$, not $\epsilon_{F}$. The cloud has range $\xi^{\prime}=v_{f} / V a$ and contains $\rho_{0}$ $V a \ll 1$ electrons. The screening cloud picture that applies to an impurity, therefore, does not apply to the lattice, and it is not necessary to screen the $f$ spins individually to have a nonmagnetic heavy fermion ground state. We have also computed the full dynamic susceptibility $\chi(q, \omega)$. We find that for $\omega<\epsilon_{f}$ this has the form appropriate to a Fermi liquid of mass $m^{*}$ and Fermi temperature $\epsilon_{f} \cdot \chi$ is determined (up to terms of relative order $m / m^{*}$ ) by the $f$-electron spins only. Our result for $\chi$ shows that the $f$ spins are delocalized at times long compared to $\epsilon_{f}^{-1}$, so that it is unnecessary to form a singlet on every site.

Next, we discuss the conductivity $\sigma(\omega, T)$ for $\omega, T<\epsilon_{f}$. We compute $\sigma$ in the usual way from the current-current response function. Our current operator is $J(q)=\Sigma_{k m}(\mathrm{k} / m) c_{k+q m}^{+} c_{k m}$, because the $f$ electrons are dispersionless and our hybridization $V$ is structureless. We assume weak disorder on the $c$-electron sites, arguing that this will not affect the mean-field equations ( 3 ) because the essential $c$-electron quantity that enters there is the density of states, which is unaffected by weak disorder. Thus we add to $H$ an impurity potential of strength $V_{i}$ which couples only to $c$-electron operators. To leading order in $1 / N$ no boson operators contribute. Applying standard impurity-averaging techniques to our model we find

$$
\sigma(\omega)=\frac{n e^{2}}{m^{*}} \frac{\tau_{i}^{*}}{1+\omega^{2} r_{i}^{*}} .
$$

Here, $\tau_{i}^{*}=\left(m^{*} / m\right) \tau_{i}$ and $\tau_{i}$ is the standard impurity scattering time; $r_{i}^{* 1}=2 \pi \rho_{0} V_{i}^{2} \cdot n$ is the density of electrons ( $c$ plus $f)$. Thus the de conductivity $\sigma(\omega=0)=n e^{2} \tau_{i} / m$ is that of a normal metal, but the frequency variation of $\sigma$ is very rapid indeed, as it is controlled by $1 / \tau_{i}^{*}$ not $1 / \tau_{i}$. Electron-boson scattering will also make a (frequency- and temperature-dependent) contribution to $\tau_{i}^{*}$. Estimating this contribuiton from the imaginary part of the one-boson term in the $c$-electron self-energy, which we find to be

$$
\operatorname{Im} \Sigma(k, \omega)(1 / N) \epsilon_{f}\left(\omega / \epsilon_{f}\right)^{2}
$$

for $\omega \ll \epsilon_{f}$ and assuming that scattering mechanisms add in parallel, we find, to leading order in $\omega^{2}$ and $T^{2}(\beta$ is a number of order 1):

$$
\frac{1}{\tau(\omega, T)}=\frac{1}{\tau_{i}}+\frac{\beta m^{*}\left(\omega^{2}+T^{2}\right)}{N m \epsilon_{f}}
$$

Substituting this into Eq. (4) at $T=0$ yields the result shown in Fig. 2. The qualitative agreement with recent experiment ${ }^{14}$ is interesting. We also find that the electron-boson scattering rate is large (compared to $\omega$ ) for $\omega=2 V a$; the direct gap shown in Fig. 1 at $k=k_{h}$ will therefore not appear as a sharp feature in $\sigma(\omega)$. This treatment is only valid if $\epsilon_{f} \gg 1 / \tau_{i}^{*} \gg \omega_{c}$, where $\omega_{c}$ is a frequency below which our treatment of electron-boson scattering breaks down.

In the $\omega=0, T>0$ case one finds for the resistivity $\rho=\sigma^{* 1}$ an equation of the form $\rho=A+B T^{2}$ where $B$, the coefficient of the $T^{2}$ term is given by

$$
B\left(\mu \Omega \mathrm{cm} / \mathbb{K}^{2}\right)=\frac{1}{N} \frac{m^{*}}{m} \frac{1}{7 n_{22} \epsilon_{f}} .
$$

Here $n_{22}$ is the carrier density in units of $10^{22}$ particles/ $\mathrm{cm}^{3}$, and $\epsilon_{f}$ is to be measured in degrees kelvin. For CePd one finds from experimental measurements ${ }^{14}$ and Eq. (6) that $B=6 / N \times 10^{-2}$ while the measured value is $12 \times 10^{-2} \cdot{ }^{15}$ Umklapp scattering, details of Fermi surface geometry, etc. have been neglected, so that one cannot expect Eq. (6) to hold precisely. In other "heavier" heavy fermion materials, $B$ is much larger. It would be interesting to see if $\mathrm{Eq} .(6)$ is correct even in order of magnitude.

Now consider the density-density correlation function $S(q, \omega)$. We have computed this in the "Fermi liquid" limit $q, \omega \rightarrow 0$ with $y=\omega / v_{F}^{*} q$ arbitrary. $\left(v_{F}^{*}=k_{F} / m^{*}\right.$ is the veloc. ity of the heavy quasiparticles.) We find that to leading order in $N, S(q, \omega)$ may be written in the familiar Landau form:

$$
S(q, \omega)=N \rho \frac{g(y)}{1+F_{0}^{s} g(y)}
$$

where $\rho=\left(m^{*} / m\right) \rho_{0}$ is the enhanced density of states,

$$
g(y)=1+\frac{y}{2} \ln \left|\frac{1-y}{1+y}\right|+i \pi y \theta(|y|-1)
$$

is the usual polarization bubble, and the Landau parameter $F_{0}^{s}=m^{*} / m$ in the Kondo limit. ${ }^{4}$ Note that all of the other Landau parameters are of order $1 / N$. The largeness of $F_{0}^{s}$ is a result of the strong constraint of $f$ occupancy expressed by Eq. (1). Thus in particular, the compressibility,

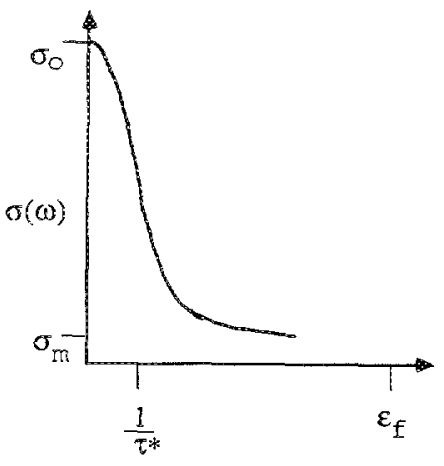

FIG. 2. Sketch of proposed form for the frequency-dependent conductivity $\sigma(\omega)$. Here $\sigma_{0}=n e^{2} \pi / m$ and $\sigma_{M} n e^{2} / m W$. Here $W$ is of order the $c$-electron bandwidti. $\sigma_{0}$ is a typical good metallic conductivity; $\sigma_{M}$ is very small, of order the Ioffe-Regel limit. 


$$
\frac{d n}{d \mu}=\lim _{q \rightarrow 0} S(q, \omega=0)
$$

is not renormalized from $\rho_{0}$, the $c$-electron value. Note also that Eq. (7) implies the existence of a zero sound mode with velocity $c$ given by $c^{2}=v_{F}^{* 2} F_{0}^{2} / 3$; this zero sound mode also appears as a singularity in the boson propagator. We consider it unlikely, however, that this mode is of physical significance, for if the long-range part of the Coulomb interaction (which on physical grounds must be present) is included in $H$ via a term.

$$
H_{c}=\sum_{q} \frac{4 \pi e^{2}}{N q^{2}} n_{q} n_{-q}
$$

(where $n$ is the total density operator and the factor of $N$ is necessary for a smooth $N \rightarrow \infty$ limit, since the charge density is define 3 to be of order $N$ ) a derivation closely paralleing that used in the familiar electron-phonon problem shows that in the electron-slave-boson case both the zero sound mode and the $q_{s} \omega \rightarrow 0$ singularity in the boson propagator are eliminated. The zero sound mode appears instead at the heavy fermion plasma frequency ${ }^{16} \omega_{p}^{*} \cong \sqrt{G} T_{k}$; while any smail $q$ singularity in the boson propagator must occur at a frequency $\omega>\epsilon_{f}$. The fact that the long-range part of the Coulomb interaction destroys the sof mode in the boson propagator has to do with the fact that the boson is a mathematical representation of an electron-electron interaction, and unlike a phonon does not represent a physical excitation of the system. This point of view has been elegantly exploited by Auerbach and Levin, ${ }^{7}$ who, working in the radial gauge, have identified the diagram in which two electrons exchange a boson with the Landau interaction function $f_{p, p^{\prime}}$. This useful identification permits one to exploit the results of Fermi liquid theory. Note that $f_{p, p^{\prime}}$, which is not itself a physical quantity, would be infrared divergent if computed in the Cartesian formulation. One may wonder about the physical significance of an interaction function which exists only in one gauge. We find, however, that one obtains the same results by explicit computation of physical quantities ${ }^{4}$ in the Cartesian gauge as one does via the Auerbach and Levin procedure ${ }^{7}$ in the radial gauge.

As our last example we mention the superconducting instability of the model. Now the siave bosons mediate a weak (order $1 / N$, except in the density fuctuation channel) interaction between the quasiparticles. It is well known that an arbitrarily weak attractive interaction will lead to superconductivity if there is no competing phase transition. We have shown that the electron-slave-boson interaction leads to $d$-wave superconductivity in our model. ${ }^{5}$ We essentially repeat the familiar derivation of superconductivity in the electron phonon model: we work to leading logarithmic order, computing the superconducting interaction by summing ladder diagrams in the particle-particle channel as shown in Fig. 3. The basic two-particle irreducible interaction vertex comes from the diagram in which two electrons near the Fermi surface exchange a slave boson: other, more complicated diagrams do not contribute to leading logarithmic order, or are small in the parameter $\epsilon_{f} / W{ }^{16}$ By performing this calculation we have found that the $s-$ and $p$-channel interactions are repulsive, and the $d$-channel interaction is attractive and larger in magnitude than the higher angular momentum interactions. We conclude that the model is unstable to $d$-wave superconductivity. We have also extended the Anderson model to inciude Coulomb interactions involving the $c$ electrons; these lead in the usual way to a repulsive Coulomb pseudopotential $\mu^{*}$ which is of order 1 . However, because the quasiparticles at the Fermi surface are (to order $m^{*} / m$ ) $f$ like, $\mu^{*}$ is renormalized down by a factor of $\mathrm{m} / \mathrm{m}^{*}$. Our final $T_{c}$ equation is then ${ }^{5}$

$$
\begin{aligned}
& T_{c}=\epsilon_{f} \exp \left(-1 / \lambda_{d}\right), \\
& \lambda_{d}=0.01 \alpha / N-\left(\mathrm{m} / \mathrm{m}^{*}\right) \mu^{*} / N .
\end{aligned}
$$

Here, $\alpha=q_{0}\left(1-N a^{2}\right) / \rho_{0} W$. Note that (i) the attractive pairing interaction is specific to the lattice: in the oneimpurity model the $s$-channel interaction is repulsive; all others vanish. (ii) The coefficient of $\alpha$ is nonuniversal and depends on details of band structure, etc. (iii) Because we work only to leading logarithmic accuracy, the quantity $\epsilon_{f}$ in Eq. (8a) may be multiplied by a factor of order unity which we are unable to compute. (iv) In the familiar phonon problem the cutoffi is $\omega_{\text {Debye }}$ and arises from the frequency dependence of the phonon propagator; here the cutoff arises from the curvature of the quasiparticle bands shown in Fig. 1, as well as from the frequency structure of the boson propagators. Because we work within weak coupling expansion, the fact that the upper cut-off is the same order of magnitude as the effective Fermi temperature of the heavy fermions is not a serious complication.

Because the Coulomb interaction is multiplied by $\left(\mathrm{m} / \mathrm{m}^{*}\right)$ it is negligable in the Kondo limit $\eta \geqslant 1$, and superconductivity is possible despite the weakness of the pairing interaction. However, as $\eta \rightarrow \infty, \epsilon_{f} \rightarrow 0$ and $T_{c}$ vanishes. As one moves toward the intermediate valence regime $\eta \sim 1, \alpha$ decreases and $m / m$ increases and superconductivity is destroyed. Thus as $\eta$ decreases from $\infty, T_{c}$ must increase, pass through a maximum at some $\eta=\eta_{\max }$, and then decrease. It seems possible to vary $\eta$ experimentally by applying pressure, $P$. In $\mathrm{Ce}$ and $\mathrm{U}$ compounds, $d n / d P<0$. In $\mathrm{CeCu}_{2} \mathrm{Si}_{2}$, $T_{c}(P)$ has the qualitative behavior expected if $\eta(P=0)>\eta_{\max }$, while in $\mathrm{UPt}_{3}$ and $\mathrm{UBe}_{13}, T_{c}(P)$ behaves as if $\eta(P=0)>\eta_{\max }$. A quantitative fit of our formula for $T_{c}(P)$ to experimental data is complicated by uncertainty in the appropriate values of the parameters of our simplified model, Eq. (2), for real heavy fermion materials with complicated Fermi surfaces.

In conclusion, the slave boson method permits explicit calculation of many interesting quantities (especially dy. namic correlation functions, which are difficult to obtain by other methods), and agrees at least qualitatively with experiment in some cases. However, it involves several drastic approximations. Also, the formal status of the various treatments of the parmater $q_{0}$ is unclear. Finally, there are some problems for which it does not seern to be useful. Foremost among these is the question of the intersite spin interactions. As mentioned above, in the lattice one does not form a singlet on every $f$ site. Intersite spin interactions are therefore possible, and it has recently been argued that they are very important in understanding the heavy fermion state. ${ }^{17}$ Such interactions must contribute to the magnetic susceptibility. 
However, in the lattice the Wilson ratio $R=\chi / \gamma$ differs from the appropriately scale single impurity value only in order $1 / N^{2}$ or $q_{0} / N^{4,7}$ Thus to study intersite spin interactions one must either work to order $1 / N^{2}$ or study the "'generalized Anderson model" in which $q_{0}$ is arbitrary. The former approach would involve a prohibitively large amount of effort; in the latter case the relation of the model studied to the physical model is unclear.

We thank N. Read and B. G. Kotliar for useful conversations. This research has been supported by the National Science Foundation under Grant No. DMR-8521377, an AT\&T Bell Labs Ph. D. Scholarship (for A.J.M.), and an NSF-CNRS Exchange Award (for M.L.).

'P. A. Lee, T. M. Rice, J. W. Serene, L. J. Sham, and J. W. Wilkins, Comm. Condensed Matter Phys. 12B, 99 (1986).

${ }^{2}$ P. Coleman, Phys. Rev B 29, 3035 (1984).

${ }^{3}$ N. Read and D. M. Newns, J. Pliys. C 16, 3273 (1983).
${ }^{4}$ A. J. Millis and P. A. Lee, Phys. Rev. B (in press); A. J. Millis, Ph.D. thesis, M.I.T. (1986).

${ }^{5}$ M. Lavagna, A. J. Millis, and P. A. Lee, Phys. Rev. Iett. 58, 266 (1987).

${ }^{6}$ N. Read, D. M. Newns, and S. Doniach, Phys. Rev, B 30, 384 (1984).

${ }^{7}$ A. Auerbach and K. Levin, Phys. Rev. Eett. $\$ 7,877$ (1986).

${ }^{8}$ 3. Rasul and H. U. Desgranges, J. Phys. C 29, L671 (1986).

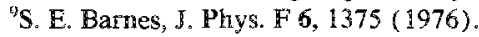

"One may also include crystal field splitting in this scheme, but we have not considered this in any detail.

"N. E. Bickers, D. L. Cox, and J. W. Wilkins, Phys. Rev. Lett. 54, 238 (1985).

12N. Read, J. Phys. C 18, 2651 (1985).

${ }^{13}$ P. Nozieres, Ann. Phys. Fr. 10, 19 (1985).

${ }^{14}$ B. C. Webb, A. J. Sievers, and T. Mihatisin, Phys. Rev. Lett. 57, 1951 (1986).

${ }^{15}$ J. M. Lawrence, J. D. Thompson, and Y. Y. Chen, Phys. Rev. Lett. 54, 2537 (1985).

itA. J. Millis, M. Lavagna, and P. A. Lee (unpublished).

${ }^{17} \mathrm{G}$. Aeppii, H. Yoshizawa, Y. Endoh, E. Bucher, J. Hufnagel, Y. Onuki, and T. Komatsubara, Phys. Rev. Lett. 57, 122 (1986); B. A. Jones and C. M. Vama, Phys. Rev. Lett. 58, 843 (1987). 